\title{
Effects of drive level on cue utilization of spatially separated redundant relevant cues*
}

\author{
JEROME S. COHEN and BRIAN SULLIVAN \\ University of Windsor, Windsor 11, Ontario, Canada
}

Albino rats trained under either a moderate or high water-deprivation level did not differ in their acquisition rates of a simultaneous brightness discrimination task in which central and peripheral cues were present. Test trials, in which only one of the set of cues was present or in which the position of the cues was reversed, revealed that moderately deprived rats were more distracted from the central cues than were highly deprived rats. Type of cue redundancy was also found to effect distraction from the central cues.

Tolman (1948) and Easterbrook (1959) maintain that drive is inversely related to cue utilization. Confirmation of this proposed relationship has been found with rats under two levels of water deprivation. Higher drive has resulted in a decreased ability for rats to utilize redundant relevant size and shape (Cohen, Stettner, \& Michael, 1969) or orientation and brightness cues (Telegdy \& Cohen, 1971) in discrimination tasks. The present study is an extension of this previous research. In the above animal studies, both relevant redundant cues occupied the same space. In the present study, redundant brightness cues occupied distinctly different areas, the side walls and the stimulus goal chamber doors of the two-choice discrimination box. It was expected that rats under high (HI) water-deprivation conditions $(23.5 \mathrm{~h})$ would be less able to utilize both redundant cues than would moderately (MOD) thirsty $(18 \mathrm{~h}$ ) animals. Furthermore, based on Solley's (1969) report of constricted peripheral field of attention in high-drive (thirst and anxiety) humans, it was expected that HI-drive rats would specifically attend less to the side-wall cues.

A further investigation involved the effect of similarity of redundant peripheral and central cues on cue utilization. Within each drive-level group, half the rats were presented the same brightness cue on each accompanying side wall and door. The remaining animals received redundant relevant cues of opposite brightness on each accompanying door and wall.

\section{METHOD}

\section{Subjects}

Thirty-two male albino rats (Wistar strain), approximately 80 days old at time of taming and shaping, were purchased from Woodlyn Farms, Guelph, Ontario. One animal failed to acquire the initial discrimination and was replaced by a substitute rat from the same breeding stock.

\section{Apparatus}

A two-choice discrimination box, as previously described in

*The following research was conducted under a grant from the National Research Council of Canada (APA 7450).
Telegdy \& Cohen (1971), was employed in the present study. Basically, the apparatus consisted of a start chamber that led into a decision chamber which faced a wall containing two $10 \times 10 \mathrm{~cm}$ interchangeable stimulus doors. By pushing open these doors, an animal could enter a goal chamber to secure liquid reinforcement (10\% sucrose solution). The decision chamber contained two side walls that radiated out from the start chamber to the edge of the wall containing the doors.

\section{Procedure}

A 15-day taming and training procedure to train the rat to open the stimulus doors for a 10-sec reinforcement, as described in the previous study (Cohen \& Telegdy, 1970), was utilized. All animals were kept on a daily 21 -h water-deprivation schedule, with $3 \mathrm{~h}$ water allowed after completing their running session during this time. The deprivation was considered appropriate for the animal to acquire the door-pushing instrumental response but sufficiently different from either high or moderate schedule. Throughout the study, each rat was run for one trial, then replaced in its home cage to wait until the rest of the animals were run before its next trial. Twelve such spaced trials were run each day. Under this procedure, average intertrial intervals were 4 min.

\section{Discrimination Training}

After acquiring a habit of opening the doors for reinforcement, animals were assigned randomly to one of two deprivation schedules and given discrimination training with redundant door and side-wall cues. Animals under high-drive (HI) conditions were deprived of water for $23.5 \mathrm{~h}$ per day and allowed to drink for only $0.5 \mathrm{~h}$ in their home cages after their daily running session. Animals under moderate-drive (MOD) conditions were deprived for $18 \mathrm{~h}$ per day and allowed $6 \mathrm{~h}$ water ad lib in their home cages after their daily running session. Food was given to all rats ad lib in their home cages.

The apparatus contained a black and white door and a $10-\mathrm{cm}$-high black or white stripe along each radiating side wall. Type of positive wall and door cue was counterbalanced within each drive-level group. For ease of explication, however, only groups in which the black door cue was positive will be described. Within each drive group, animals were assigned randomly to the same or opposite redundant brightness cue group. For the same group, the positive door stimulus was identical to the brightness of stripe along the door's appositional wall. The same, of course, was true for the negative door and wall stimuli. In this group, an animal would receive reinforcement if it ran along the black striped wall and opened the black door. For the opposite group, the positive door stimulus was opposite in brightness to its appositional wall. The same condition held for the negative door. Thus, in approaching the positive black door, an animal had to run on the same side as the white striped wall. Thus the study contained four drive-cue redundancy subgroups, each containing eight animals: HI-same, HI-opposite, MOD-same, MOD-opposite.

All animals were given 12 self-corrected trials per day. The position of the negative and positive redundant cues were varied in a random sequence, as described by Fellows (1967). The incorrect door was always locked, and an error was considered to have been made when an animal touched that door. Criterion for learning the brightness discrimination was considered when a rat made no more than two errors within two consecutive trial blocks (24 trials).

\section{Testing}

This phase was designed to determine the differences in 
Table 1

Mean Errors to Learn the Original Discrimination Task (Standard Deviations are in Parentheses)

\begin{tabular}{lcccr}
\hline & \multicolumn{3}{c}{ Side Wall } \\
\cline { 2 - 5 } Drive & \multicolumn{2}{c}{ Same } & \multicolumn{2}{c}{ Opposite } \\
\hline HI & 28 & $(10.3)$ & 37 & $(14.3)$ \\
MOD & 30 & $(13.1)$ & 26 & $(8.8)$ \\
\hline
\end{tabular}

distraction from stimulus change and amount of cue utilization of wall and door cues between the drive groups. Eight test days were employed. On each test day, 12 spaced trials were presented. Every fourth trial was a test trial, while intertest trials were the same as those presented during the original discrimination training. On each test day, the rat was presented three types of test trials designed to test for utilization of door cues (door test), wall cues (wall test), and strength of stimulus control by both cues in a rearranged manner (switch test). During each test trial, both doors were unlocked and any choice was reinforced. A similar procedure was used by Sutherland \& Holgate (1966) to prevent test trials from becoming specific discrimination learning trials for the rat. Below is an outline of each test trial when the black door was positive.

Door Test. Only the doors contained the brightness stimuli, the side walls being solid gray. For either opposite or same groups, choice of the black door was counted as correct door response; choice of the white door was counted as an error.

Wall Test. The doors were both gray, while the side contained the brightness stimuli. Choice of a door on the same side as the black striped wall was a correct response for a same group animal. Choice of the door on the same side as the white striped wall was the correct choice for an opposite group animal.

Switch Test. In this test, the relative strength of the door and wall cues in eliciting the approach response was determined. Here the door and wall cues were switched from the positions used in the original discrimination. In the same group, a white striped side wall now accompanied a black door and a black striped wall now accompanied a white door. In the opposite group, the accompanying wall and doors contained the same brightness cues. Logically, an animal could not make an error on a switch test trial, since an approach to a negative door also meant an approach to the side of the positive side wall. In view of this situation, only door choices were recorded for these trials. Order of the daily three test trials were randomized so that each test was presented in every temporal order at least twice.

If a rat committed more than 2 errors per day or more than 14 errors on the 72 regular intertest discrimination trials, it was dropped from the study and another animal was substituted for it. This procedure insured that test trial errors were not the result of general errors during the test phase. One animal had to be replaced for failure to meet this criterion.

\section{RESULTS AND DISCUSSION}

As seen in Table 1, drive level of type of cue redundancy did not affect initial rate of acquisition. All groups appeared to learn the original discrimination within the similar number of errors. Analysis of variance failed to show significant main or interaction effects. Individual comparisons between groups did not reveal any significant differences.

During the test phase, very few errors on the test trials were made. Only five animals made more than four errors on any one test. These few animals were distributed equally over groups. Since only eight test trials were given for each test, too few errors could have been made to analyze group data by parametric analysis. Instead, a criterion for cue utilization based on binomial probabilities was used in determining if a rat had utilized a door or wall cue. If an animal made no more than one error on the wall or door test trials, it was considered to have used the door or wall cues, respectively, beyond .05 probability of chance. An animal was considered to have utilized the door cues if it had made more than one error on either the door test or switch test trials (for doors). An animal was considered a wall-cue utilizer, then, if it made no more than one error on either the wall test or the switch test (for walls) trials. An animal was considered to have utilized both door and wall cues if it reached this criterion on both door and wall or switch test trials for each spatially located cue. All two-cue utilizers reached criterion only on doors and wall test trials.

As seen in Table 2, very few animals within any of the groups were able to utilize both wall and door cues. No significant drive or cue redundancy group differences were found for number of two-cue utilizers. For the single cue utilizers, door cues were more salient in that a significant proportion of animals utilized them rather than the wall cues $(p<.01)$. Drive level or cue redundancy failed to affect the saliency of the single cue used.

These findings are contrary to expectations derived from the drive level span of attention hypothesis advanced by Tolman (1948) and Easterbrook (1959). Although MOD-drive animals did not show more cue utilization, they appeared to be more distracted by test stimulus change conditions during testing. Whereas all HI-drive animals showed at least utilization of one cue, only 11 out of 16 MOD animals were able to demonstrate any sort of cue utilization, a nonsignificant proportion $(p=.10)$. Due to the fact that none of the single-cue utilizers under MOD drive used wall cues, the data suggest that the peripheral wall changes distracted such animals from correctly employing door cues rather than vice versa.

Switch test trials (Table 3) further revealed that HIdrive animals were less distracted from the use of the door cues than MOD-drive Ss. A significant proportion of HI-drive animals, 13 out of 16 , correctly utilized the door stimuli $(p=.01)$, while only half of the MOD

Table 2

Number of Rats in Each Group $(\mathbf{N}=8)$ Utilizing One, Two, or No Cues (Number of Door Utilizers in the One-Cue Category is in Parentheses)

\begin{tabular}{llccc}
\hline \multicolumn{1}{c}{ Group } & $\begin{array}{l}\text { One } \\
\text { Cue }\end{array}$ & $\begin{array}{l}\text { Two } \\
\text { Cue }\end{array}$ & $\begin{array}{c}\text { No } \\
\text { Cue }\end{array}$ \\
\hline HI-Same & 6 & $(6)$ & 2 & - \\
HI-Opposite & 6 & $(4)$ & 2 & - \\
MOD-Same & 4 & $(4)$ & 2 & 2 \\
MOD-Opposite & $4(4)$ & 1 & 3 \\
\hline
\end{tabular}


animals were able to do so. Examination of the effect of type of cue redundancy during the initial discrimination training revealed that the opposite-trained animals were more distracted than the same-trained animals during the switch test trials. A significant proportion of same animals, 13 out of 16 , correctly utilized door cues $(p=.01)$, while only half of the opposite-trained rats were able to do so. For individual drive groups, the HI-same group was the least distracted and the MOD-opposite group was the most distracted. For only HI-same animals, significantly more rats utilized the door cues than not $(p=.035)$. HI-same snimals significantly differed from MOD-opposite animals in the proportion of rats utilizing the central door cues ( $p \leqslant .05$, Fisher test).

These results suggest that peripheral wall cues, when attended to at all, served to operate as context stimuli rather than information or discrimination stimuli. If the latter were the case, then opposite-trained animals would have acquired the original discrimination more slowly due to a more complex task of conflicting compound brightness stimuli. This clearly did not happen. The greater disruption seen for wall switches in the opposite group further supports the interpretation that the wall stimuli served as surround stimuli. That is, the switching of wall stimuli for opposite animals appears to have decreased the distinctiveness of the door cues. The less distraction to HI-drive animals by wall cue changes also supports the notion that increased drive focuses or narrows the range of attention. As Easterbrook (1959) has stated, an increased field of attention due to decreased drive can also have a disorganizing effect on cue utilization, i.e., producing competing orienting responses to the reinforced response. The present study suggests that spacially distinct redundant cues will be
Table 3

Number of Rats in Each Group $(\mathbf{N}=8)$ Utilizing Door or Wall Cue on Switch Test Trials

\begin{tabular}{lcc}
\hline & \multicolumn{2}{c}{ Switch } \\
\cline { 2 - 3 } Group & Door & Wall \\
\hline HI-Same & 7 & 1 \\
HI-Opposite & 6 & - \\
MOD-Same & 6 & - \\
MOD-Opposite & 2 & - \\
\hline
\end{tabular}

more noticed by organisms operating under lower drive but will not be used as relevant cues for reinforcement-contingent behavior. Previous work in the present laboratory suggests that redundant cues occupying the same physical space will be more utilized as relevant by animals under lower drive conditions.

\section{REFERENCES}

Cohen, J. S Stettner, L. J \& Michael, D. J. Effect on deprivation level on span of attention in a multi-dimension discrimination task. Psychonomic Science, 1969, 15, 31-32.

Cohen, J. S., \& Telegdy, G. A. Effect of drive level on habit strength in a discrimination task. Psychonomic Science, 1970, 19, 27-29.

Easterbrook, J. A. The effect of emotion on cue utilization and the organization of behavior. Psychological Review, 1959, 66, 183-201.

Fellows, J. Chance stimulus sequences for discrimination tasks. Psyshological Bulletin, 1967, 67, 87-92.

Solley, C. M. Effects of stress on perceptual attention. In B. P Rourke (Ed.), Explorations in the psychology of stress and anxiety. Don Mills: Longmans Canada, 1969. Pp. 1-14.

Sutherland, N. S., \& Holgate, V. Two cue discrimination learning in rats. Journal of Comparative \& Physiological Psychology, 1966, 61, 198-207.

Telegdy, G. A., \& Cohen, J. S. Cue utilization and drive level in albino rats. Journal of Comparative \& Physiological Psychology, 1971, 75, 248-253.

Tolman, E. C. Cognitive maps in rats and men. Psychological Review, 1948, 55, 189-208.

(Received for publication March 19, 1973.)

\section{Latency patterns in category judgments*}

\section{FRANK D. McGUIRK and JOHN A. HÉBERT \\ Colorado State University, Fort Collins, Colo. 80521}

Adult human Ss were required to learn a conditional discrimination in which there were three distinct visual stimuli (squares) to be matched with three corresponding response buttons. When Ss had learned the task to criterion, they were presented with a test series of 11 stimuli, including, and spaced symmetrically around, the three training stimuli. Ss were told to place each successive stimulus in one of the three categories represented by the choice of three response buttons. Response latency and category choice were recorded. The prediction that Ss

*Material from this report was presented at the Psychonomic Society meetings in St. Louis, November 1971. would respond most quickly to stimuli at both ends of the series, and that the stimuli in the middle of the center category would elicit more rapid responses than those that were between the categories, was confirmed.

In recent years, there have been a number of adaptation level (AL) studies reporting, among other measures, response latencies. In 1968, Capehart and Pease conducted a study in which the latency pattern was one of the dependent variables of interest. Briefly, their study involved a two-category judgment task, in which Ss were to make light or heavy responses to each weight in a series that followed experience with a "neutral" training weight. They found a significant stimulus effect on the latency, or decision time, measure, with a tendency for short latencies 